\title{
Midtrimester genetic amniocentesis in Eastern Ontario: a review from 1970 to 1985
}

\author{
ALASDAIR G W HUNTER, DIANE THOMPSON, AND \\ MARSHA SPEEVAK \\ From the Division of Genetics, Children's Hospital of Eastern Ontario, Ottawa, Canada KIH 8LI.
}

SUMMARY Greater use of genetic amniocentesis in the Eastern Ontario region occurred once the results from the various national trials were published. ${ }^{1-3}$ Acceptance also paralleled an increase in the number of low parity older women having children and the centralisation and greater publicity given to our programme in the late 1970s. A centralised approach has had the positive effects of preventing unnecessary procedures, assuring appropriate patient counselling, follow up, and review, and of increasing obstetric and laboratory expertise. Advanced maternal age has been largely responsible for the increased demand for the service and accounted for an increasing proportion of tests performed, while the absolute number for several other diagnostic categories remained unchanged. We found no evidence that women with a history of previous miscarriage had a higher rate of pregnancy loss following the procedure, and comparison with a group of women who declined amniocentesis did not show that the test increased the risk of miscarriage.

Genetic amniocentesis was first offered in Ottawa in 1970 . From then until the end of 1985,4084 women contacted the Division of Genetics and 3275 of them underwent the procedure. By 1 January 1979 over 20 obstetricians had performed a total of 370 tests, and the early cases were contributed to the Canadian Collaborative Study. ${ }^{1}$ In 1979 area obstetricians agreed to have five of their colleagues (in three hospitals) perform all amniocenteses and to have the counselling and record keeping centralised through the Division of Genetics at the Children's Hospital of Eastern Ontario. The aim was to ensure that patients made informed decisions about amniocentesis, that the guidelines on indications were followed, and that obstetric expertise was maximised. In January 1983 the laboratory changed from flask subcultures to in situ colonial analysis. This reduced the rate of reported mosaicism, increased the case load handled per technologist, and decreased the turn around time for samples. In 1985 there was renewed interest in the safety, efficacy, and acceptability of genetic amniocentesis because of the growing medical promotion and public acceptance of chorionic villus sampling, with which it is compared. We therefore thought this an opportune time to review our experience with amniocentesis.

Received for publication 15 April 1986 Accepted for publication 30 May 1986.

\section{Methods}

Only three amniocenteses were carried out in Ottawa before the beginning of the Canadian MRC study in January 1972, which introduced specific data collection forms and the requirement that patients be followed up. The cytogenetic laboratory continued to use these forms after the study ended in 1975, although the data were not always complete. After the programme was centralised in 1979 , all procedures were booked through the Division of Genetics and most patients were formally counselled there. New forms were introduced which allowed easy entry of data into a dBase II computer file. One of the participating obstetricians continued to do his own counselling for advanced maternal age (AMA) but used the same forms. Data routinely collected included: gestation at visit and at tap, by dates and by ultrasound, and at delivery; age at delivery, past pregnancy, and family history, and reason for the procedure; and recent radiation, drug, alcohol and smoking history, type of counselling, and $\mathrm{Rh}$ status. In addition, information as to whether a tap was attempted, the number of insertions required, the quality and quantity of fluid obtained, placental location, and various laboratory procedures were recorded. Follow up included standard information on pregnancy outcome and complications, birth weight, and the attitude of the patient to the 
procedure. The reason why a patient who had been counselled declined the test was noted, as was the discovery of a dead fetus at the time of the tap. Starting in 1982, a record was kept of women who declined amniocentesis and counselling when first contacted for an appointment. Cases were summarised and reviewed twice a year. A concerted effort was made at the time of each review to update follow up information, and separate attempts have been made on several occasions to find missing data, including during the course of this review.

Data were analysed using the standard logical search formats of dBase II as well as command programmes normally used for an annual review of the outcome of patients referred to the prenatal programme. For purposes of several comparisons, the data have been divided into pre-1979, 1979 to 1982 , and 1983 to 1985 . At the time of writing many patients tapped in 1985 were yet to deliver, and so data concerning miscarriage risks and follow up end on 31 December 1984.

\section{Results}

DEMOGRAPHIC TRENDS

Fig 1 shows, by year, the number of women giving birth at or over the age of 35 years (AMA) in ou catchment area, which has about 15000 deliveries year. It also gives the number and percentage of AMA women who underwent the procedure, and finally the total number of women per year wh $\vec{\phi}$ underwent amniocentesis. Genetic amniocentesis was introduced at a time when the age specifiö fertility of women of 35 and over was falling. rapidly. ${ }^{4}$ Only a small fraction of these women had amniocentesis. The numbers began to show as marked rise in 1976 to 1977 and this coincided wit the publication of a number of surveys attesting tou the relative safety of the test, ${ }^{1-3}$ as well as the centralisation of the programme in mid-1979. How ever, more important was the fact that a cohort of educated women, who had postponed their families began to have children in large numbers. Thus,

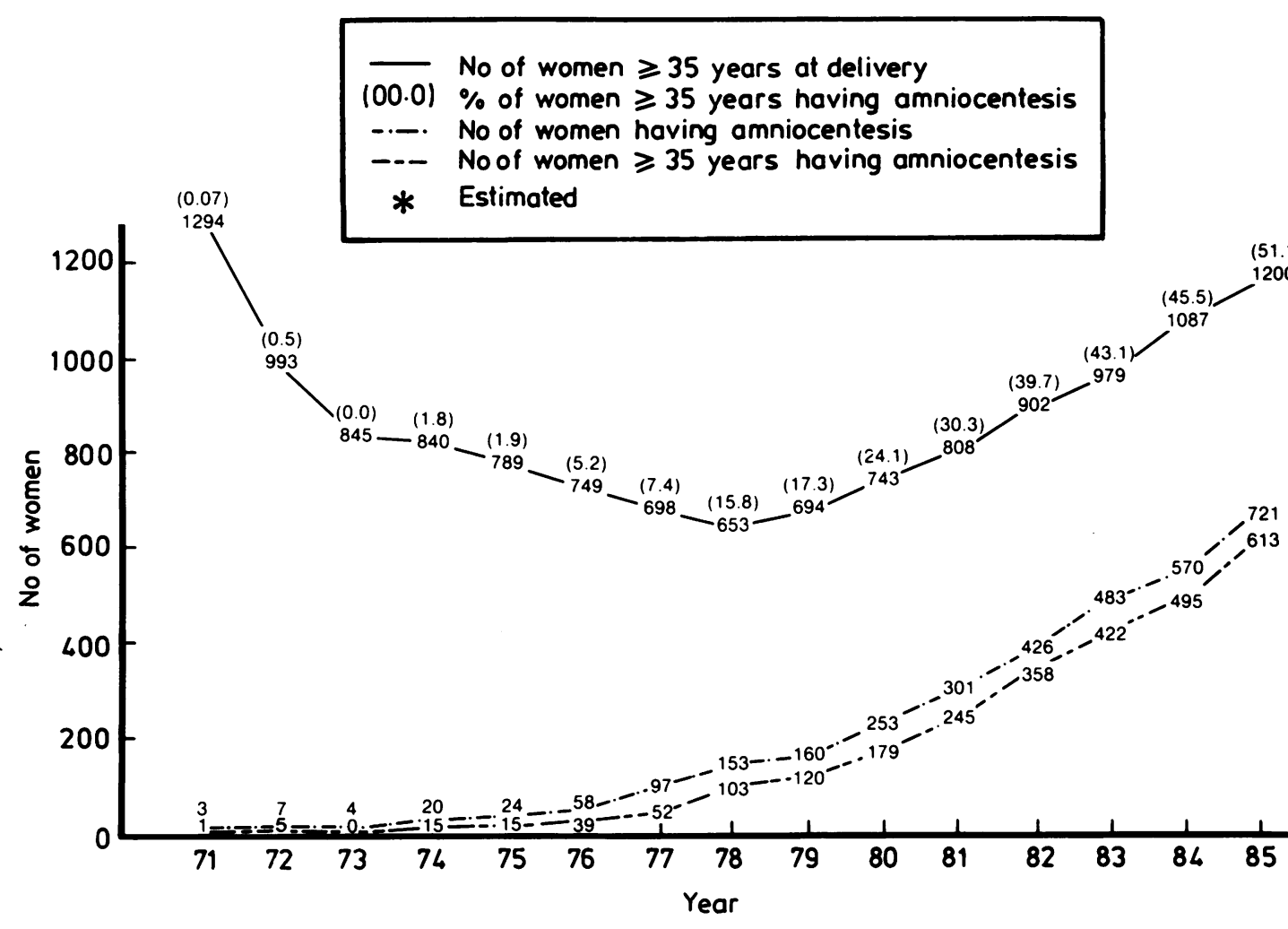

FIG 1 No of women giving birth at or over 35 years by year. 
some extent, the increase in amniocenteses performed paralleled the rising age specific fertility of women over 35 . The percent usage has also increased steadily to the point that in 1985 we estimate that just over $50 \%$ of AMA women had an amniocentesis and that about $70 \%$ were in contact with our department.

The data have been divided into the three time periods previously described so as to provide a number of comparisons: pre-1979, 1979 to 1982, and 1983 to 1985 . Table 1 summarises the gravid status of all women referred to the programme and provides a separate tabulation for those referred for AMA. For all periods, and regardless of indication, almost half the amniocenteses were performed during a first or second pregnancy. We had anticipated this would be the case for the latter years but had expected a higher proportion of multigravid women before 1979. For example, in 1970 , only $8.8 \%$ of Ontario women delivering over the age of 35 were primiparas, and in 1977 the figure was $16 \%{ }^{+}$By contrast, $29 \cdot 8 \%$ of our pre-1979 AMA mothers were gravida 1 (the percentage who would be para 1 would be even higher). Thus it appears that even before 1979 a large proportion of AMA women using amniocentesis represented women who had postponed their families and therefore were of low gravidity. Women of high gravidity represented a small group with low utilisation throughout the period of study. The fact that the proportion of gravida 1 women was higher before 1979 than after suggests that the pre- 1979 period was the beginning of the 'career delayed baby boom'.

Although advanced maternal age has always been the most common indication for genetic amniocentesis, its relative dominance has continued to increase over the years from $60 \%$ before 1979 to its current level of $85 \%$ (table 2). Initially, women who had already had an abnormal child were proportionately more likely to be tested and the actual number of tests performed for such indications has remained relatively constant, with the exception of a decline in testing for neural tube defects in second degree relatives (largely replaced by ultrasound and serum $\alpha$ fetoprotein screening). Few women in our region have had amniocentesis for fetal sexing because they were at risk of transmitting an X linked disease. The minimum $50 \%$ probability that a male would be unaffected seemed to be the major deterent Tests performed for inappropriate reasons were eliminated once the programme was centralised in 1979.

The age distribution of patients has paralleled the shift in the indications. Mean maternal age was $34 \cdot 1$ years before $1979,35.8$ in the period 1979 to 1982 .

TABLE 1 Gravid status of women referred for amniocentesis $(\%)$

\begin{tabular}{|c|c|c|c|c|c|c|}
\hline & \multicolumn{3}{|c|}{ All women } & \multicolumn{3}{|c|}{ AMA women } \\
\hline & $\operatorname{Pre}-1979$ & $1978-82$ & $198.3-85$ & Pre'-1979 & $1979-82$ & $198.3-8.5$ \\
\hline Gravida 2 & $26 \cdot(1)$ & $27 \cdot 5$ & $29 \cdot 4$ & $20 \cdot 6$ & $27 \cdot 6$ & $29 \cdot 4$ \\
\hline Gravida 3 & $26 \cdot 8$ & $26 \cdot(1)$ & $25 \cdot 1$ & $23 \cdot 4$ & 25.7 & $25 \cdot 8$ \\
\hline Gravida 4 & $11 \cdot 7$ & $15 \cdot 0$ & $14 \cdot 4$ & $12 \cdot 4$ & $15 \cdot 2$ & $14 \cdot 5$ \\
\hline Gravida 5 & $6 \cdot 6$ & $5 \cdot 5$ & $6 \cdot 2$ & $101 \cdot 1$ & $5 \cdot 8$ & $6 \cdot 4$ \\
\hline Gravida $>7$ & $1 \cdot 4$ & 1.7 & $2 \cdot()$ & 0.9 & $1 \cdot 9$ & $2 \cdot 3$ \\
\hline Total on which pereentage based & 366 & 1340 & $2(147$ & 218 & 1165 & 17165 \\
\hline
\end{tabular}

$x^{2}=2014$, df $5 . p<0.1015$ for 1979 to 1982 versus pre-1979 comparison of gravid status. (Gravida 6 and 7 combined because of small numbers expected.)

TABLE 2 Indications where genetic amniocentesis performed.

\begin{tabular}{|c|c|c|c|c|c|c|}
\hline & \multicolumn{2}{|c|}{$\operatorname{Pre}-1974$} & \multicolumn{2}{|c|}{$1979-42$} & \multicolumn{2}{|c|}{$1983-85$} \\
\hline & No & $\%$ & No & "。 & $N_{0}$ & $\%$ \\
\hline Maternal age $\geqslant 35$ y at delivery & 218 & $6(1) \cdot 6$ & 949 & $s(1.4$ & 1487 & $86 \cdot()$ \\
\hline First degrec relative with NTIS & 45 & $12 \cdot 6$ & 62 & 5.3 & 56 & $3 \cdot 2$ \\
\hline Second degree relative with NTD & 13 & 3.6 & 44 & 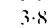 & 22 & 1.3 \\
\hline Fetal sex for $X$ linked disease & 3 & $(1 \cdot 5$ & 9 & 0.8 & 2 & 0.1 \\
\hline Enzymopaths & 2 & 0.6 & 4 & 0.3 & 6 & 0.3 \\
\hline Previous trisomic intant & .37 & 10.3 & 51 & 4.4 & 40 & $2 \cdot 3$ \\
\hline Parental translocation & 4 & $1 \cdot 1$ & 9 & 0.8 & 5 & 0.3 \\
\hline Hacmoglobinopathy & 0 & (1.) & 4 & 0.3 & 4 & 0.2 \\
\hline Anxicty (maternal age 3.3-34) & 7 & $2 \cdot 11$ & $2 x$ & $2 \cdot 4$ & $\overrightarrow{79}$ & $4 \cdot 6$ \\
\hline Abnormality suspected on U/S & 3 & 0.8 & 7 & $(1) \cdot 6$ & 17 & $1 \cdot()$ \\
\hline Other specific indication & i) & 0.0 & 0 & 0.0 & 9 & $(1) .5$ \\
\hline No indication for test & 27 & $7 \cdot 5$ & 6 & 0.5 & (1) & 0.0 \\
\hline Total number of women in groups & 3711 & & 1176 & & 1729 & \\
\hline
\end{tabular}




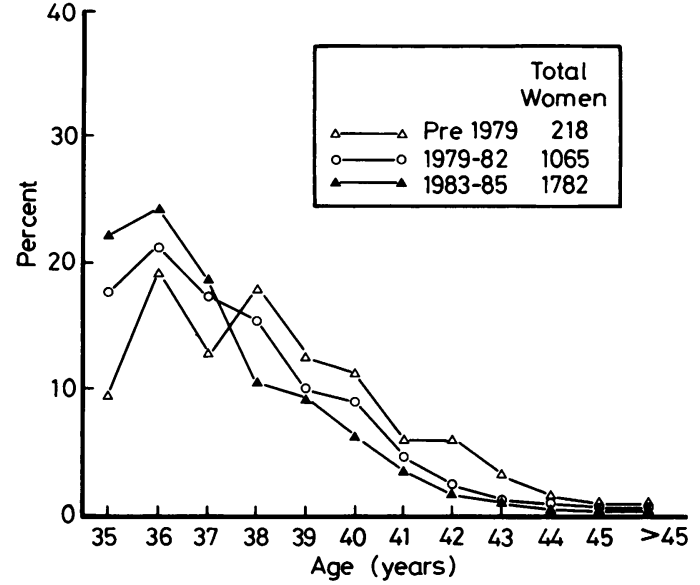

FIG 2 Age distribution of women referred for $A M A(\%)$.

and 35.9 in 1983 to 1985 . Before $1979,37.9 \%$ of women were under 35 , in 1979 to 1982 the figure was $18.2 \%$ and in 1983 to 1985 it was $16 \%$. Fig 2 shows the trend towards younger women within the AMA group which has an impact on the cost benefit ratio, both with respect to any inherent risks of the procedure and the rate of detection of chromosome anomalies (see below).

COUNSELLING

A major rationale for centralising the regional amniocentesis programme was to ensure that all patients received complete and standardised counselling, in order that they might make an informed decision about amniocentesis. Table 3 provides a breakdown of how patients proceeded through the programme during the three phases. Before 1979 only $7 \cdot 4 \%$ of women received formal counselling while the remainder received highly variable in formation from the more than 20 obstetricians, wh each carried out relatively few procedures. By 1983 to $1985,92 \%$ of women referred, and $98.5 \%$ of thos proceeding to amniocentesis, had received forma counselling through the programme, although some times circumstances dictated that this be done bis telephone.

Centralisation has increased the completeness of follow up of pregnancy outcome. This was recorded in $76 \%$ of pre-1979 cases (despite several retro. spective attempts at follow up), in $95.7 \%$ from 1978 to 1982 , and in $98.2 \%$ from 1983 to 1984 (1985 is dif necessity incomplete). At present, incomplete follow up tends to be limited to women who deliver outsides our catchment area.

It has never been our intention to carry out $\$$ formal study of patients' attitudes to amniocentesis but since 1980 all women have been asked to return follow up questionnaire and $804(\sim 25 \%)$ have don so. Of this number, 792 were either satisfied or very satisfied, while 10 were either dissatisfied or ver®o dissatisfied. Of the latter 10 , four were counselled by the participating obstetrician $(1.1$ expected), thres complained of inadequate information, and one fe she had been coerced into having the test by he⿳亠丷厂 doctor. The remaining six $(8.9$ expected) werö counselled within the genetics division; one ha\& chronic amniotic fluid leak and a premature labour one had a failed culture, one felt the wait for the result was too long, one was concerned about the needle and had concern about the possible need fợ. abortion, two gave no reason, and one felt the counselling approach was wrong. Nine women sai they would not repeat the procedure $(1 \cdot 1 \%)$ and sis

TABLE 3 Counselling profiles of patients.

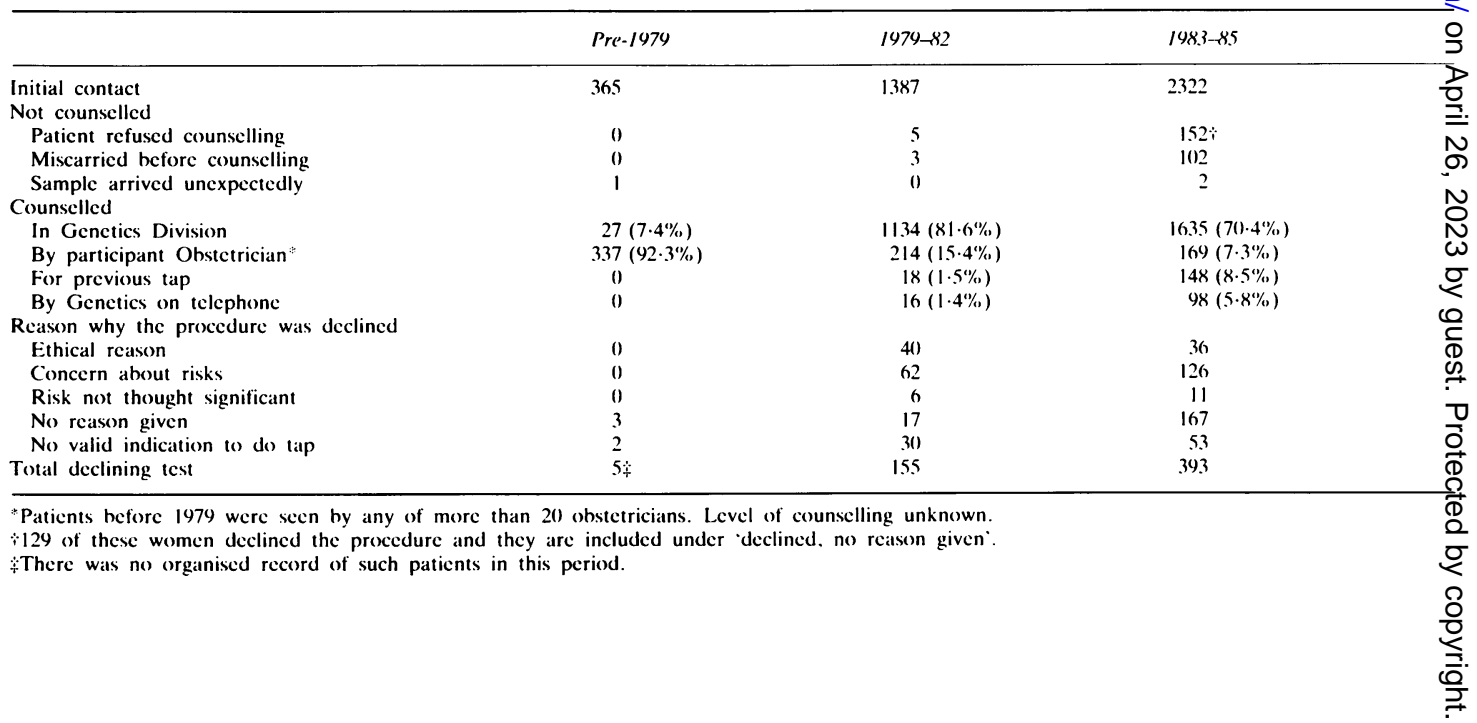


of these were from the dissatisfied group. One of the remaining three was concerned about fetal risk and two gave no reason. In total, 733 women expressed no concerns, 26 commented on the length of time required for results and three about the possible need for abortion, 24 felt some aspect of the test had been inadequately explained, while two thought there had been too much explanation. We think that women with definite complaints would be as likely to respond as those who had none, so that in general there seems to be a high level of satisfaction with the programme.

With time there was an increasing trend for husbands to accompany their wives for counselling: $22 \cdot 2 \%$ were seen as couples before $1979,52 \cdot 3 \%$ from 1979 to 1982 , and $59 \%$ from 1983 to 1985 . Anecdotally, we have noted that many women who decline counselling appear to experience significant anxiety, and they continually phone for results and with questions.

The percentage of women who declined the procedure despite an appropriate indication, and who did not miscarry before the test, was $16.9 \%$ in the period 1983 to 1985 (table 3). We did not keep data on women who declined the test at the time of initial telephone contact until 1982, and so the "no stated reason' group is smaller before 1983. However, it is apparent that concern about possible risks of the test is the most commonly stated reason that patients actually referred to the service give for declining the test. The average age of women who declined on the basis of perceived risk from the test was 35.24 years, as compared to $36 \cdot 14$ in women who declined for ethical reasons.

\section{OBSTETRICAL FACTORS}

A further reason for centralising the programme was the belief that limiting the procedure to a smaller number of obstetricians would increase individual expertise, minimise complications, and maximise compliance with the established guidelines and our standard record keeping. Table 4 demonstrates a steady increase in the overall success of the procedure.

Another measure of increasing facility with the technique is the fact that before 1979 fluid was obtained with a single insertion in only $76 \cdot 3 \%$ of cases. By 1979 to 1982 the figure was $89 \cdot 3 \%$, and by 1983 to 1985 it was $94 \cdot 3 \%$. Similarly, in 1979 to 1982 , both members of nine of 15 twin pairs were successfully tapped $(64 \cdot 3 \%)$, as compared to 15 of 18 $(83 \cdot 3 \%)$ from 1983 to 1985.

Some may consider that a $2.6 \%$ initial failure to obtain fluid at amniocentesis (table 4) is high for current practice. However, this rate is mainly due to a conservative approach to using more than one needle insertion. For example, in the pre-1979 and 1979 to 1982 phases, $42 \%$ of tap failures had only a single needle insertion, and this figure was $69 \%$ in 1983 to 1985 . Undoubtedly the failure rate would be reduced by performing multiple insertions. A concern about these initial tap failures is that 12 of $45(26 \cdot 7 \%)$ of those women in the 1983 to 1985 period did not return for a repeat tap and therefore did not obrain a result. The women who did not return did not differ from the total group, or from those who did return, with respect to maternal age or indication, and they did not differ from those returning with respect to the number of initial insertions. This suggests that women who undergo amniocentesis retain significant ambivalence, perhaps with respect to safety, and that they are easily deterred when things do not go smoothly on the first attempt.

Based on the 2645 procedures where information on placental location was recorded, $35 \cdot 4$ posterior, 17.2 anterior (transected by the needle), and 18.4 anterior (not transected) placentas were expected among the 71 tap failures where placental location was adequately documented. However, 25,25 , and 21 respectively were observed $\left(\chi^{2} 13 \cdot 9, p<0 \cdot 005\right)$, suggesting that anterior placenta was a risk factor for tap failure.

TABLE 4 Rate of success in obtaining results from genetic amniocentesis.

\begin{tabular}{|c|c|c|c|}
\hline & Pre-1979 & $1979-82$ & $1983-85$ \\
\hline \multicolumn{4}{|l|}{ Initial procedure } \\
\hline Initial sampling atttempt made & 370 & 1176 & 1729 \\
\hline Initial failure to obtain fluid & $13(3 \cdot 5 \%)$ & $31(2 \cdot 6 \%)$ & $45(2 \cdot 6 \%)$ \\
\hline Initial culture failure & $12(3 \cdot 2 \%)$ & $4(0 \cdot 3 \%)$ & $1(0 \cdot 05 \%)$ \\
\hline No karyotype attempted & 2 & 9 & 2 \\
\hline \multicolumn{4}{|l|}{ Second attempt } \\
\hline Failed again to obtain fluid & 8 & 2 & 1 \\
\hline No second attempt made & 10 & 11 & 12 \\
\hline Culture from second tap failed & 1 & () & 0 \\
\hline Total women not obtaining a result & $19(5 \cdot 1 \%)$ & $13(1 \cdot 1 \%)$ & $13(0 \cdot 75 \%)$ \\
\hline
\end{tabular}




\section{LA B O R A TOR Y}

Successful culture of amniotic fluid by the laboratory has become a virtual certainty. During the 1983 to 1985 period, the single failure in 2063 attempts occurred with a sample of only $3 \mathrm{cc}$ of fluid. Table 5 summarises the abnormalities detected through the programme and the outcome of those pregnancies. Most women elected to terminate their pregnancy when a significant abnormality was detected, and most families appear to consider Turner's syndrome significant, despite what we would consider an optimistic counselling approach. On the other hand families decided to continue two of six pregnancies with Klinefelter's syndrome and both with $47, X Y Y$.

Until 1983 the laboratory used the flask method of culture and mosaicism that was significant enough to discuss with the parents occurred in nine of 1426 $(0.63 \%)$ of cases and resulted in two terminations (despite our counsel of a probable normal outcome), neither of which could be confirmed on post-abortion tissue. Following institution of the in situ colonial method this occurred in only three of $1715(0 \cdot 17 \%)$ cases and resulted in the termination of one pregnancy where the presence of the marker had been confirmed from a fetal cord sample at 20 weeks' gestation.

A second advantage of the in situ method has been with respect to laboratory efficiency. In 1981, using the flask method, two technologists carried out 291 fluid analyses (145 each) with an average time fro receipt of sample to written report of 24.35 days. 1985, $3 \cdot 5$ technologists complete 697 tests (199 eaclo with an average turn around of 17.5 days.

The age specific rates of the major trisomies amon women who did not present because of previous trisomy, or because an abnormality had been sus pected on ultrasound, were $0 / 405$ under $35 ; 6 / 9$ ஓ $(0.006)$ at 35 to $36 ; 10 / 1001(0.010)$ at 37 to 39 , and $5 / 319(0.016)$ at or over 40 years at deliver Klinefelter's syndrome or 47,XYY occurred $1 / 40$. under $35,4 / 991$ at 35 to $36,2 / 1001$ at 37 to 39 , an $2 / 319$ at or above 40 at delivery. Two of the 'Turneris syndrome' were found in women under 35 , one at $38_{0}$ and one at 40. Down's syndrome occurred in orie (aged 30) of 128 women tested because of a history of a previous trisomic livebirth. The highest rate $f$ trisomy detected was in those pregnancies where ant abnormality was suspected from a previous ultre sound examination $(4 / 27)$.

\section{RATES OF PREGNANCY LOSS}

As already mentioned, the risk of losing the pregnancy was a major concern for women considering amniocentesis and those that had already had miscarriage often expressed concern that they migh be at higher risk. Therefore, we have looked at feta

TABLE 5 Abnormal results obtained.

\begin{tabular}{|c|c|c|c|c|c|c|}
\hline & \multicolumn{5}{|l|}{ Outcome } & \multirow{2}{*}{$\frac{\text { 吾 }}{\underline{\underline{3}}}$} \\
\hline & Termination & Carried to term & Miscarried & Stillbirth & Total & \\
\hline \multicolumn{6}{|l|}{ Chromosomal } & \\
\hline Trisomy 21 & 19 & 0 & 1 & 0 & 20 & $\underline{\sigma}$ \\
\hline Trisomy 18 & 2 & $1^{*}$ & ) & $2(U)$ & 5 & 그. \\
\hline Trisomy 13 & $1(U)$ & 0 & 1 & 0 & 2 & . \\
\hline $47 . X X Y$ & 4 & 2 & 0 & 0 & 6 & \\
\hline 46. XY (X linked) & 2 & 1 & 0 & 0 & 3 & \\
\hline $45 . \mathrm{X}$ & $4 \div$ & 0 & 0 & 0 & 4 & \\
\hline $5 p-$ & 2 & 0 & 0 & 0 & 2 & \\
\hline $9 p-$ & 1 & 0 & 0 & 0 & 1 & \\
\hline \multicolumn{7}{|c|}{ Normal cell line with minor mosaic abnormal cell line } \\
\hline \multicolumn{7}{|c|}{ Pre-1983 conversion to in situ colonial method: based on 1426 results } \\
\hline+20 & 1 & 3 & 0 & 0 & 4 & \\
\hline+13 & 1 & 0 & () & 0 & 1 & \\
\hline Iso $(X)$ & () & 1 & () & 0 & 1 & \\
\hline Marker & 0 & 1 & 0 & 0 & 1 & \\
\hline & & & & & Total 9 & S \\
\hline \multicolumn{7}{|c|}{ After conversion to colonial method: based on 1715 results } \\
\hline+20 & 0 & 1 & 0 & 0 & 1 & \\
\hline+8 & () & 1 & 0 & () & 1 & $\bar{D}$ \\
\hline Marker & $1 \$$ & 0 & 0 & () & 1 & \\
\hline
\end{tabular}

Also detected and leading to termination: CNS anomalies 12, $\alpha$ thalassaemia 3. $\beta$ thalassaemia 1. lactic acidosis 1, MPS IIIa 1, Potter syndrome 2, omphalocele $\Phi$ *Trisomy 18 to term. no karyotype done on fluid.

tOne mosaic 47,XYY, 46, XY

†One 45.X 46,X.iso(X).

\$Present in fetal cord sample.

(U)Suspected on ultrasound. 
loss in a number of ways. Fig 3 summarsies total pregnancy loss by age and previous miscarriage history for all women, including those who declined the test. Induced abortions were excluded, but losses before and after the procedure were included. As expected, miscarriage rates increased with maternal age, regardless of previous miscarriage history. The pregnancy loss rates did not differ significantly between women who had 0 , one, or two previous miscarriages. Numbers were small, but women who had had more than two previous miscarriages did show a higher overall loss rate. Three year moving averages have been graphed in order to minimise random fluctuations due to the small numbers in each age group. The loss rates observed were $123 / 1829(6.7 \%)$ for women with no previous loss, $28 / 484(5.7 \%)$ for those with one previous loss, $8 / 142$ $(5 \cdot 6 \%)$ for those with two previous losses, and $9 / 62$ $(14.5 \%)$ for those with more than two losses. Comparing the total loss rate for women with no previous loss to that for women with more than two losses gave a $\chi^{2}$ of $5.59(p>0.025)$.

A question often asked by patients and physicians is whether or not a woman with a history of previous miscarriage has a higher risk from amniocentesis than a woman who has no such history. Table 6 compares the rates of post tap loss in patients with 0 , one, and two or more previous pregnancy losses. Although the latter groups are small there is no evidence that a previous history of pregnancy wastage increases the likelihood of postamniocentesis miscarriage.

The important question as to whether or not amniocentesis increases the rate of pregnancy loss is more difficult to address. Those cases where spontaneous pregnancy loss occurred were studied by gestation at loss and were compared between women who underwent amniocentesis and those who declined the procedure. There were 72 miscarriages that occurred before the patient could be counselled; 46 were before 12 weeks and in 26 the gestation was not recorded but was early. Data on miscarriages occurring after 12 weeks are summarised in table 6 .

Amniocentesis patients have an ultrasound at the time of the procedure and non-viable pregnancies are detected, whereas such losses will occur spontaneously and later in the 'no tap' group. It is therefore more valid to compare pregnancy loss from 12 weeks until term in the two groups. Losses at unknown gestation are excluded. This gives 14/350 $(3.9 \%)$ in the 'no tap' and $79 / 2110(3.7 \%)$ in the 'tap' group. The first post tap loss occurred at 17 weeks. The loss rate in the 17 to 20 week period was $21 / 2071$ $(1.01 \%)$ for the 'tap' and $5 / 346(1.44 \%)$ for the 'no tap' cases, and so there was no evidence of a cluster of losses in the immediate post-tap period. Indeed the loss rates are the same in all gestational intervals.

We have tried to examine whether the outcome of an index pregnancy influences the likelihood that women will return for a subsequent amniocentesis.

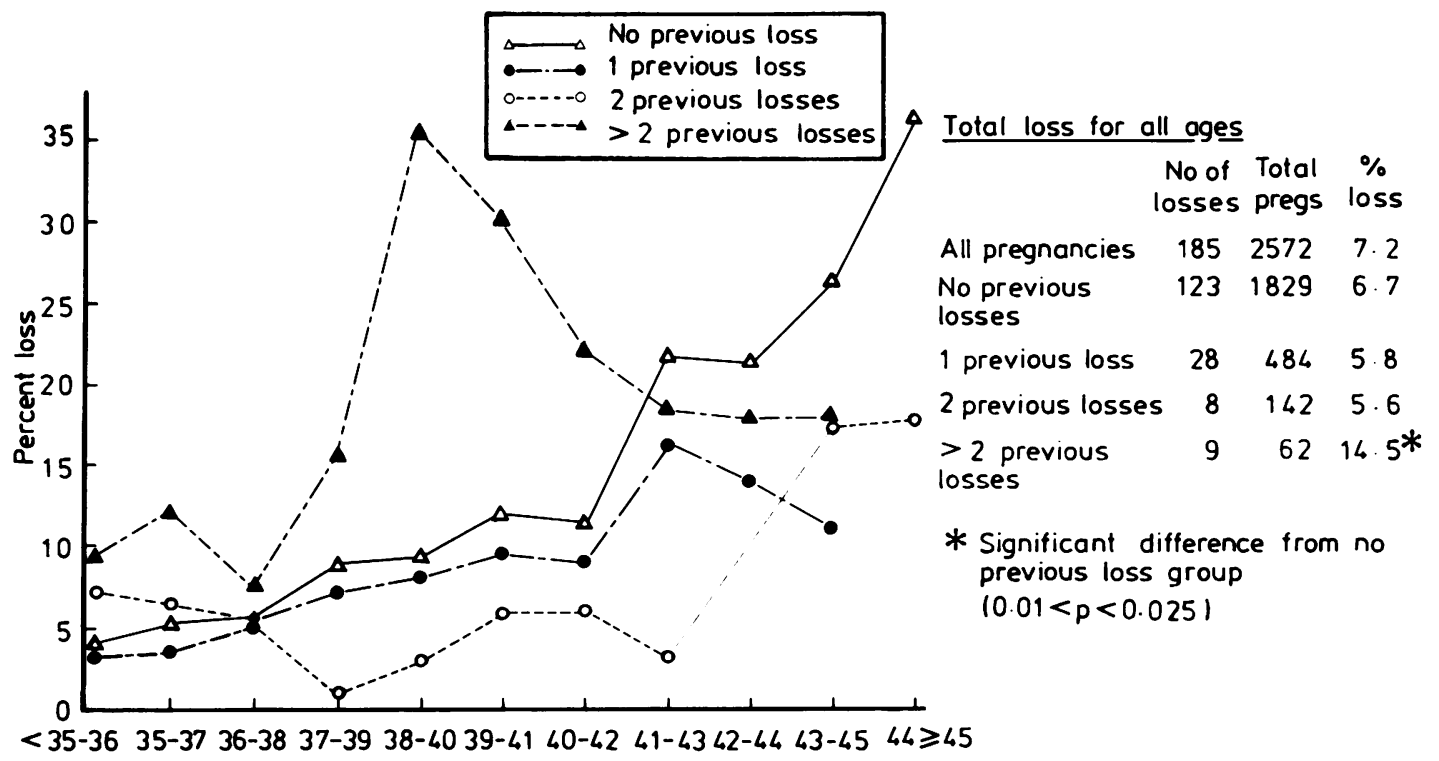

FIG 3 Total fetal loss in pregnancy compared by age and previous history of pregnancy wastage. 
TABLE 6 Rate of pregnancy loss after amniocentesis by previous loss history.

\begin{tabular}{|c|c|c|c|c|c|c|c|}
\hline \multirow{2}{*}{$\begin{array}{l}\text { No of previous } \\
\text { miscarriages }\end{array}$} & \multicolumn{2}{|c|}{ Miscarriage } & \multicolumn{2}{|c|}{ Stillbirth } & \multicolumn{2}{|c|}{ Total loss } & \multirow{2}{*}{$\begin{array}{l}\text { Total group } \\
95 \% \text { confidence } \\
\text { intervals }\end{array}$} \\
\hline & No & Rate & No & Rate & No & Rate & \\
\hline 0 & $18 / 1494$ & 0.012 & $7 / 1494$ & 0.005 & $25 / 1494$ & 0.017 & $(0 \cdot(011-(0 \cdot() 23)$ \\
\hline 1 & $7 / 395$ & 0.018 & $1 / 395$ & 0.003 & $8 / 395$ & 0.02 & $(0) \cdot(0) 7-(0) \cdot(0.33)$ \\
\hline$>2$ & $1 / 161$ & 0.006 & $1 / 161$ & $0 \cdot 006$ & $2 / 161$ & 0.012 & $(0 \cdot 0(0) 4-0 \cdot(020)$ \\
\hline
\end{tabular}

Rate of pregnancy loss in amniocentesis patients vs those declining.

\begin{tabular}{|c|c|c|c|c|c|c|}
\hline \multirow[t]{3}{*}{ Gestation } & \multicolumn{2}{|c|}{ No amniocentesis (351) } & \multicolumn{4}{|c|}{ Amniocentesis (2155) } \\
\hline & \multirow[t]{2}{*}{ No } & \multirow[t]{2}{*}{$\%$} & \multicolumn{2}{|c|}{ Before tap } & \multicolumn{2}{|c|}{ After tap } \\
\hline & & & No & $\%$ & No & $\%$ \\
\hline $12-<20$ weeks & 10 & $(2 \cdot 85)^{*}$ & 50 & $(2 \cdot 32)$ & 10 & $(0 \cdot 46)$ \\
\hline $20-<28$ weeks & 2 & $(0.58)$ & - & & 12 & $(0.59)$ \\
\hline 28 -term & 2 & $(0.59)$ & - & & 7 & $(0.35)$ \\
\hline 17-20 weeks & 5 & $(1 \cdot 44)$ & - & & 21 & $(1 \cdot 01)$ \\
\hline $12-28$ weeks & 12 & $(3 \cdot 44)$ & - & & 72 & $(3.45)$ \\
\hline 12 -term & 14 & $(4 \cdot 01)$ & - & & 79 & $(3 \cdot 78)$ \\
\hline
\end{tabular}

*All denominators are corrected for loss before that time.

Overall, 197 of $3275(6 \%)$ procedures and $6.36 \%$ of women were repeaters. Of these women, $109(3.5 \%)$ were returning after a successfully completed pregnancy. Assuming that $85 \%$ of the 72 women who miscarried before being counselled would have had the test, then 15 of $110(13.6 \%)$ women who miscarried before a planned amniocentesis returned for a tap with a subsequent pregnancy. This compares with four of $79(5 \%)$ women who miscarried after amniocentesis and eight of $38(21 \%)$ who had an induced abortion because of a fetal abnormality. If women who miscarry before a planned test are considered as a rough baseline as to the likelihood of a subsequent pregnancy, then there is evidence that women attribute post-amniocentesis loss to the procedure and are less likely to return. On the other hand, those who terminate an affected pregnancy have a relatively higher rate of return.

\section{Discussion}

We have used a commercial software package to maintain our clinical records since 1980 and believe this has had a positive effect upon the quality of the service and the usefulness of the data in our files. Furthermore, routine statistical summaries and research projects have been greatly facilitated. A standard multisheet form was also developed which reduced the work required to ensure that complete and standardised information was recorded, and about 30 minutes is required daily to enter the data for the more than 1000 patients now being referred each year.

The trend to increased utilisation of amniocentesis is universal and was recently summarised by Baird $e \overrightarrow{0}$ $a l .{ }^{5}$ Although they do not specifically comment, their. $=$ data also show the trend to increasing numbers of women over 35 giving birth. This fact, as much as the greater rate of utilisation, has had a major impact on the need to provide resources for genetic amnio centesis. It is not known how long this trend to delayed families will continue, but it is probably reasonable to predict that the rapid increases in demand will end once the 'baby boom' has passed? through the 35 and 45 age group. There is nothing ye to suggest a plateau in our rate of utilisation.

Our data on gravid status and specific indications follow logically from the increasing numbers of older $r_{O}$ mothers who have delayed their families. The initiaBㅡ. amniocentesis survey ${ }^{2}{ }^{3}$ collected data in the earlys 1970s and had a significantly lower rate of gravida 13 women than in our review. Even among our womeno seen before $1976,22 \cdot 4 \%$ were gravida 1 .

There are a variety of organisational structures whereby prenatal diagnosis services can be delivered All aspects of the procedure in Ontario are covered $N$ by the universal health care system. The centralised approach has assured that a high level of counselling is provided to the patient, patient satisfaction is high $\omega$ there is good communication between geneticists and obstetricians, procedures are not performed fof inappropriate reasons, and standardised data are⿻ maintained which allow for periodic reviews of quality control and statistics. A further advantage is that the laboratory is always aware when a specimend is expected, can plan their workload, and, im- $\frac{-}{\circ}$ portantly, can immediately start enquiries if a sample fails to arrive. 
Our laboratory has had several years experience with both the flask subculture method and in situ colonial analysis. ${ }^{67}$ As discussed, we believe the latter method has advantages with respect to distinguishing pseudomosaicism and to the number of samples that can be handled per technologist.

As expected, the overall rate of pregnancy loss from the time of referral until delivery increased with maternal age. ${ }^{8}$ Notably, there was no significant difference in rate for women who had 0 , one, or two previous losses, but the rate was higher for those with more than two losses. This is compatible with the data of Warburton and Fraser. ${ }^{9}$ However, contrary to the recent report by Esrig and Leonardi, ${ }^{10}$ we did not find evidence that post-amniocentesis losses were more common in women with a history of miscarriage. The loss rates in the two studies are comparable for those women with no previous pregnancy wastage, but we failed to find a raised rate for those who had had earlier losses. The NICHD study $^{3}$ also found no difference attributable to a history of previous pregnancy loss.

The women who declined amniocentesis did not differ significantly in age or miscarriage history from those who had amniocentesis, and there was no evidence of a greater fetal loss rate in women who had the test. The loss rate from 17 weeks to term was $9 / 344(0 \cdot 026,95$ th confidence interval $0 \cdot 012$ to $0 \cdot 04)$ in the 'no tap' group, and 40/2056 (0.019, 95th confidence interval 0.013 to 0.025 ) in the 'tap' group. From 12 weeks to term the rates and confidence intervals were $0.04(0.02$ to 0.06$)$ and $0.038(0.03$ to $0.046)$ respectively.

Although we could not show an increased loss rate after amniocentesis, our results suggest that women do associate the procedure with any subsequent spontaneous loss and are less likely to return for another amniocentesis. This is not the case for induced abortion for fetal abnormality.
We would like to thank our many obstetric colleagues who co-operated in the organisation of this programme, especially Drs M Gillieson, P Hall, G. Hulley, B Ivey, and H Muggah. We are also grateful to ultrasound technologists S Sklar, K McAuliffe, M Cusson, S Gaudette, and C Desjardins at the Ottawa Civic and General Hospitals.

\section{References}

'Medical Research Council of Canada (MRC). Diagnosis of genetic discase by amniocentesis during the second trimester of pregnancy. MRC Report No. 5. 1977.

2 Medical Research Council. Working party on amniocentesis report. An assessment of the hazards of amniocentesis. $\mathrm{Br} J$ Obstet Gynaecol 1978:85(suppl 2):1-41.

${ }^{3}$ NICHD National Registry for Amniocentesis Study Group. Midtrimester amniocentesis for prenatal diagnosis. Safety and accuracy. JAMA 1976;236:1471-6.

+ Statistics Canada: Census of Canada. Births. Annual Bulletin 92-723 (1969-1983).

5 Baird PA. Sadovick AD. McGillivray BC. Temporal changes in the utilization of amniocentesis for prenatal diagnosis by women of advanced maternal age. Prenat Diag 1985:5:191-8.

- Cox DM, Niewczas-Late V. Riffell MI. Hamerton JL. Chromosomal mosaicism in diagnostic amniotic fluid cell cultures. Pediatr Res 1974:8:679-83.

7 Brierly K. Speevak M. Chromosome preparations from amniotic fluid cell culture in 7-10 days. Ann Meeting Cytogenet Technol, Tuscon. Arizona (Abstract). 1983.

${ }^{x}$ World Health Organization. Spontaneous and induced abortion. Tech Rep Ser 461.1970.

${ }^{y}$ Warburton D. Fraser FC. Spontancous abortion risks in man. Data from reproductive histories collected in a medical genetics unit. Am J Med Genet 1964:16:1-25.

10 Esrig SM, Leonardi DE. Spontaneous abortion after amniocentesis in women with a history of spontaneous abortion. Prenat Diag 1985;5:321-8.

Correspondence and requests for reprints to $\mathrm{Dr}$ Alasdair Hunter, Division of Genetics, Childrens' Hospital of Eastern Ontario, 401 Smyth Road, Ottawa, Ontario, Canada K1H 8LI. 